\title{
BMJ Open Predictive study of tuberculosis incidence by time series method and Elman neural network in Kashgar, China
}

\author{
Yanling Zheng (D) , ${ }^{1}$ Xueliang Zhang, ${ }^{1}$ Xijiang Wang, ${ }^{2}$ Kai Wang, ${ }^{1}$ Yan Cui ${ }^{2}$
}

To cite: Zheng $Y$, Zhang $X$, Wang $X$, et al. Predictive study of tuberculosis incidence by time series method and Elman neural network in Kashgar, China. BMJ Open 2021;11:e041040. doi:10.1136/ bmjopen-2020-041040

- Prepublication history for this paper is available online. To view these files, please visit the journal online (http://dx.doi org/10.1136/bmjopen-2020041040).

Received 30 May 2020 Revised 24 December 2020 Accepted 05 January 2021
Check for updates

(C) Author(s) (or their employer(s)) 2021. Re-use permitted under CC BY-NC. No commercial re-use. See rights and permissions. Published by BMJ.

${ }^{1}$ State Key Laboratory of Pathogenesis, Prevention and Treatment of High Incidence Diseases in Central Asia, College of Medical Engineering and Technology, Xinjiang Medical University, Urumqi, China ${ }^{2}$ Center for Disease Control and Prevention of Xinjiang Uygur Autonomous Region, Urumqi, China

Correspondence to Professor Xueliang Zhang; shuxue2456@126.com and Dr Yan Cui; cuiyan_jk@sina.com

\section{ABSTRACT}

Objectives Kashgar, located in Xinjiang, China has a high incidence of tuberculosis (TB) making prevention and control extremely difficult. In addition, there have been very few prediction studies on TB incidence here. We; therefore, considered it a high priority to do prediction analysis of TB incidence in Kashgar, and so provide a scientific reference for eventual prevention and control. Design Time series study.

Setting Kashgar, China Kashgar, China.

Methods We used a single Box-Jenkins method and a Box-Jenkins and Elman neural network (ElmanNN) hybrid method to do prediction analysis of TB incidence in Kashgar. Root mean square error (RMSE), mean absolute error (MAE) and mean absolute percentage error (MAPE) were used to measure the prediction accuracy.

Results After careful analysis, the single autoregression (AR) $(1,2,8)$ model and the AR $(1,2,8)$-ElmanNN (ARElman) hybrid model were established, and the optimal neurons value of the AR-Elman hybrid model is 6 . In the fitting dataset, the RMSE, MAE and MAPE were 6.15, 4.33 and 0.2858 , respectively, for the $\operatorname{AR}(1,2,8)$ model, and $3.78,3.38$ and 0.1837 , respectively, for the AR-Elman hybrid model. In the forecasting dataset, the RMSE, MAE and MAPE were $10.88,8.75$ and 0.2029 , respectively, for the $\operatorname{AR}(1,2,8)$ model, and 8.86, 7.29 and 0.2006, respectively, for the AR-Elman hybrid model.

Conclusions Both the single AR $(1,2,8)$ model and the AR-Elman model could be used to predict the TB incidence in Kashgar, but the modelling and validation scaledependent measures (RMSE, MAE and MAPE) in the AR (1, $2,8)$ model were inferior to those in the AR-Elman hybrid model, which indicated that the AR-Elman hybrid model was better than the AR $(1,2,8)$ model. The Box-Jenkins and ElmanNN hybrid method therefore can be highlighted in predicting the temporal trends of TB incidence in Kashgar, which may act as the potential for far-reaching implications for prevention and control of TB.

\section{INTRODUCTION}

Tuberculosis (TB) is still a major global public health problem and the ninth leading cause of death in the world. ${ }^{1-3}$ TB accounts for a large loss to society and for a significant reduction in the labour force, because (1) TB is contagious, (2) patient resistance is low, and (3) treatment time is long. All countries recognise this and are working
Strengths and limitations of this study

The Box-Jenkins method has good prediction performance and high prediction accuracy.

- Elman neural network can capture the non-linear information of time series well.

- A hybrid model often improves the prediction accuracy of a single model.

- The long-term prediction accuracy of AR-Elman hybrid model will decline.

hard to fight TB. In 2018, the number of new reported TB cases was about 10 million; this figure has remained relatively stable in recent years. The latest treatment results show that the global TB treatment success rate is $83 \%$. WHO has set targets for the 'stop TB' strategy. The targets mention that by 2030 , on the basis of the work in 2015, TB deaths should be reduced by $90 \%$, and annual new TB cases should be reduced $80 \% .^{4}$ In order to achieve these goals, TB prevention and control services must be provided in the broad context of universal health coverage, joint action must be taken to address the social and economic consequences of $\mathrm{TB}$, and technological breakthroughs should be achieved by 2025 in order to make the TB incidence decline faster than that an any time in history. According to the global TB report $2019,{ }^{4}$ China has the second highest number of TB cases in the world. ${ }^{4}$ The TB incidence in western China was much higher than that in eastern and central China. The province with the highest $\mathrm{TB}$ incidence in the west is Xinjiang province. In 2016 and 2017, the annual TB incidence per 100000 people in Xinjiang was 185.66 and 202.58, respectfully, nearly three times higher in Xinjiang than that national level in the same years.

There are 14 Prefectural-Level cities in Xinjiang, China, among which Kashgar has a very high TB incidence rate. In 2016 and 2017, the annual TB incidences per 100000 people in Kashgar were 427.44 and 465.33, 
respectively, nearly seven times higher than the national level. Doing a good job in the prevention and control of TB in Kashgar is, therefore, an important link to reduce the TB incidence in Xinjiang and China.

Mastering changing law of the incidence of infectious diseases, using the existing surveillance data to analyse, then, to predict possible epidemic trend and provide reference data for the prevention, can better help to control occurrence and epidemic of infectious diseases. Prediction of infectious diseases is to predict occurrence, development and epidemic trend of infectious diseases according to the occurrence, development law and related factors of infectious diseases.

There are many forecasting methods for infectious diseases: the grey prediction method, ${ }^{5}$ the exponential smoothing prediction method, ${ }^{6}{ }^{7}$ the dynamic model prediction method, ${ }^{8}$ the Box-Jenkins method, ${ }^{9}$ the neural network method, ${ }^{10}$ with the deepening of prediction research, more and more scholars like to use the BoxJenkins method, ${ }^{11-21}$ there are many different models in this method, and if appropriate models are chosen according to the characteristics of time series, high prediction ability often can be obtained. The Neural network has a strong nonlinear mapping ability, in which Elman neural network is composed of input layer, hidden layer, connection layer and output layer. The Elman network has a dynamic memory function, and it is very suitable for time series prediction. At present, the Elman network is widely used in various fields, and has achieved notable successful prediction results. ${ }^{22-26}$ Sometimes, the prediction effect of a single model is not ideal, in order to further improve the prediction accuracy, many studies adopt the combined model prediction method, ${ }^{27-29}$ the combined model can absorb the advantages of two or more methods so as to achieve a higher prediction accuracy.

The TB incidence in Kashgar is very high, and it is urgent to do a good job in the prevention and control of TB in this area. Accurate prediction of TB incidence is a prerequisite for prevention and control, which can help advance in resource planning and policy formulation. In this study, the popular Box-Jenkins time series method was used to build a model for predicting the TB incidence in Kashgar, and in order to improve the prediction accuracy, the Elman neural network with its strong ability to capture nonlinear information was used to construct the combined model for prediction analysis.

\section{MATERIALS AND METHODS \\ Study area and data sources}

We selected Kashgar as the study site (see figure 1). This area is located in the south of Xinjiang province in China and has an area of approximately $16.2000 \mathrm{~km}^{2}$ and a permanent population of 4.64 million in 2018. TB case data from January 2005 to December 2017 were obtained from the Center for Disease Control and Prevention (CDC) of Xinjiang Uygur Autonomous Region, all TB cases in Xinjiang must be reported to the CDC through

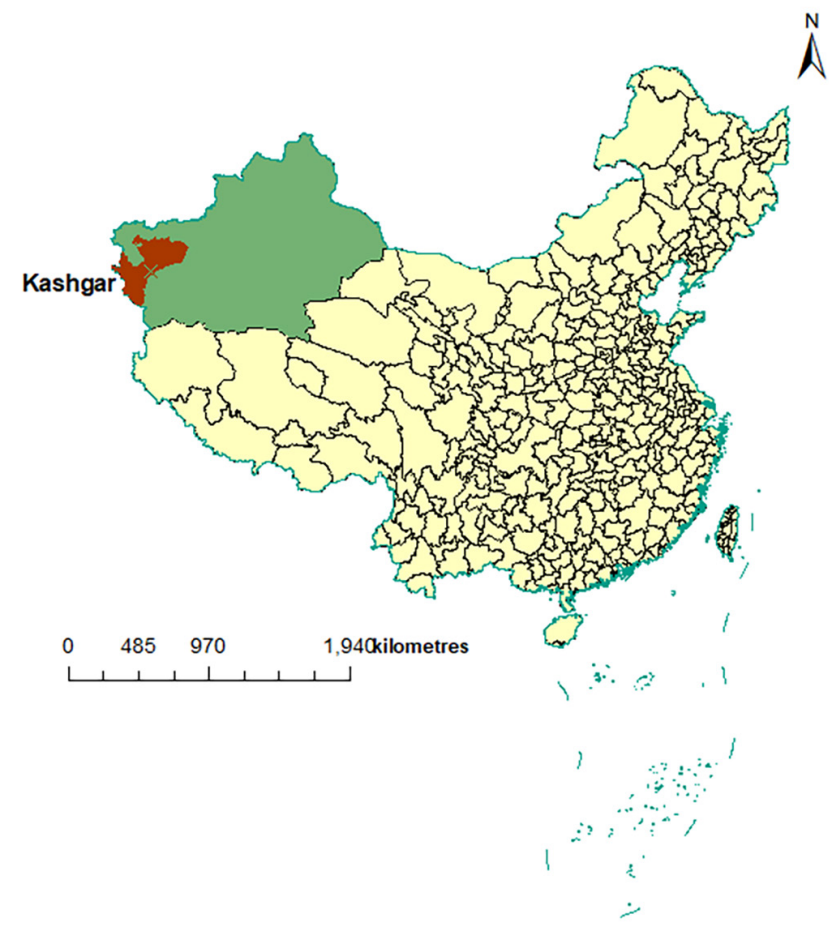

Figure 1 The red part of this picture is the location of Kashgar in Xinjiang, China. Kashgar is located in the South of Xinjiang, and it has a very high incidence of tuberculosis.

the infectious disease surveillance system within 24 hours. The TB cases datasets used need permission of the CDC. Population data were obtained from the website of Xinjiang Bureau of Statistics (http://tjj.xinjiang.gov.cn/ $\mathrm{tjj} / \mathrm{tjfw} /$ list_tjfw.shtml). Based on the population data and TB case data, we calculated the incidence data of TB.

\section{Patient and public involvement}

Patients were not involved in the design of this study as it involved only observational analysis of an anonymised, pre-existing, routinely collected dataset.

\section{Autoregressive moving average model}

The autoregressive moving average (ARMA) model $^{30}$ is an important time series analysis and prediction model in Box-Jenkins method, also known as an auto-regression moving average model. The ARMA $(p, q)$ model is a model with autocorrelation order $p$ and moving average order $\mathrm{q}$, the $\mathrm{p}$ and $\mathrm{q}$ are judged by the autocorrelation function (ACF) and partial ACF (PACF) diagram of stationary data. If the original data are stable, the autocorrelation coefficients are trailing, and the partial correlation coefficients are p-order truncated, then $q=0$, the ARMA $(p, q)$ model is written as AR (p), and its expression is as follows:

$X_{t}=c+\phi_{1} X_{t-1}+\phi_{2} X_{t-2}+\ldots+\phi_{p} X_{t-p}+\varepsilon_{t}$

where, $X_{t}$ is the observed value at $\mathrm{t}, \phi_{1}, \ldots, \phi_{p}$ are model parameters, c is a constant, if only $\phi_{1}, \phi_{2}, \phi_{p}$ are not zeros, then, The AR (p) model becomes a sparse model, which can be written as $\operatorname{AR}((1,2, p))$.

If the original data are stable, the autocorrelation coefficients are q-order truncated and the partial 
correlation coefficients are trailing, then $\mathrm{p}=0$ and the ARMA (p,q) model becomes MA (q), its expression is as follows:

$$
X_{t}=\mu+\varepsilon_{t}+\theta_{1} \varepsilon_{t-1}+\theta_{2} \varepsilon_{t-2}+\ldots+\theta_{q} \varepsilon_{t-q}
$$

where, $\mu$ is the expected value of $X_{t} \cdot \theta_{1}, \ldots, \theta_{p}$ are model parameters.

If the original data are stable, the autocorrelation coefficients are trailing, and the partial correlation coefficients are also trailing, then the expression of the ARMA (p, q) model is as follows:

$$
X_{t}=c+\phi_{1} X_{t-1}+\phi 2 X_{t-2}+\ldots+\phi_{p} X_{t-p}+\varepsilon_{t}-\theta_{1} \varepsilon_{t-1}-\theta_{2} \varepsilon_{t-2}-\ldots-\theta \varepsilon_{t-q^{*}}
$$

There are four main steps in ARMA modelling:

First step. The prerequisite for ARMA modelling is the stationary of time series. Check whether the data are stable by the Augmented Dickey-Fuller (ADF) unit root test. In this study, the significant level probability $(\mathrm{p}$ value) is 0.05 , and if the $\mathrm{p}$ value is less than 0.05 , then, the data are considered stable. By observing the ACF and the PACF of the stable data, we can determine the possible values of $p$ and $q$ and establish the possible ARMA ( $p, q$ ) model.

Second step. The parameters of ARMA (p, q) model are estimated by the maximum likelihood estimation or the least square estimation, and the model parameters are tested. If $\mathrm{p}$ value is less than 0.05 , the parameters have statistical significance. The best model is determined according to the value of the Akaike information criterion (AIC), the Schwarz criterion (SC) and the Goodness of Fit $\left(\mathrm{R}^{2}\right)$ of model. The smaller AIC and SC are, the larger the $\mathrm{R}^{2}$ is, and the better the model is.

Third step. To determine whether the established ARMA (p, q) model is suitable. The residual sequence of a suitable model shall be the white noise process, and its ACF and PACF coefficients should be within twice the SD range, otherwise, it is considered that the extraction of information in the established model is not sufficient, and it is necessary to consider improving the accuracy of the model.

Fourth step. Using the established model to do prediction and analysis.

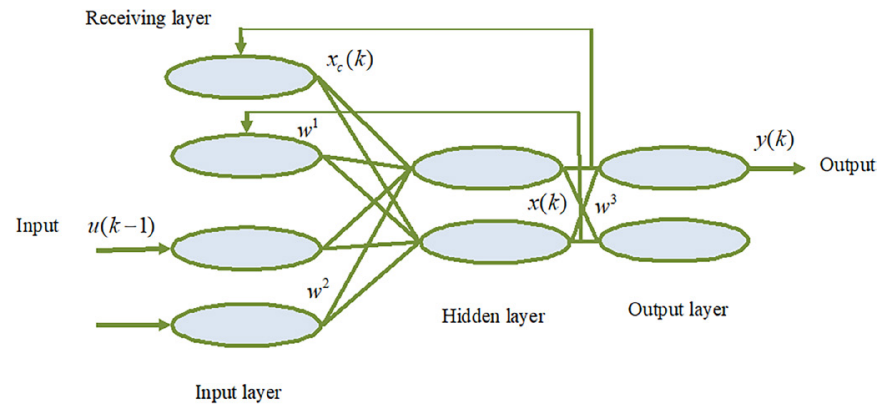

Figure 2 The structure diagram of Elman neural network. $w^{1}, w^{2}$ and $w^{3}$ are the connection weight matrixes. $x_{c}(k)$ and $x(k)$ represent the output of the contact unit and the hidden layer unit, respectively, $y(k)$ represents the output of the output unit, $u(k-1)$ represents the input of the input unit.

\section{Elman neural network model}

The E1man neural network (see figure 2) was proposed by E1man in $1990 .{ }^{31}$ The model is generally divided into four layers: input layer, hidden layer, receiving layer and output layer. The characteristic of the E1man network is that the output of the hidden layer is connected to the input of the hidden layer through the delay and storage of the receiving layer, which makes it sensitive to the data of the historical state. The addition of the internal feedback network increases the ability of the network itself to deal with dynamic information, thus achieving the purpose of dynamic modelling.

The mathematical structure of the Elman neural network is as follows:

$$
\begin{aligned}
& x(k)=f\left(w^{1} x_{c}(k)+w^{2} u(k-1)\right) \\
& x_{c}(k)=\alpha x_{(}(k-1)+x(k-1) \\
& y(k)=g\left(w^{5} x(k)\right)
\end{aligned}
$$

Where, $w^{1}$ is the connection weight matrix between the contact unit and the hidden layer unit, $w^{2}$ is the connection weight matrix between the input unit and the hidden layer unit, $w^{3}$ is the connection weight matrix between the hidden layer unit and the output unit. $x_{c}(k)$ and $x(k)$ represent the output of the contact unit and the hidden layer unit, respectively, $y(k)$ represents the output of the output unit, $\alpha$ is a self-connected feedback gain factor, $0 \leq \alpha<1, f(x)$ often takes the sigmoid function.

There are four main steps in Elman neural network modelling:

First step. Data standardisation processing. Data standardisation is scaling the data to a small specific interval. In order to remove the unit limit of the data and convert it into dimensionless pure value, it is convenient for the index of different units or order of magnitude to be compared and weighted. In our study, we used function package mapminmax () to standardise the data, standardised data were in the range ( -1 to 1$)$.

Second step. Determine the input layer, the output layer. Generally, the input and output layer are determined according to the characteristics of data and the needs of the analysis.

Third step. Set the parameters of the Elman model, such as training epochs and goals. In our study, training epochs and goals of the Elman neural network were set to 2000 and 0.00001 , respectively. Determine the number of neurons in the hidden layer, so that the error of the established Elman model is minimised. At present, there is no ideal analytical expression for the number of neurons in the hidden layer. The number of neurons has a great influence on the performance of the network. When the number of neurons is too large, it will lead to that the network learning time being too long, poor generalisation performances and even failure to converge, but when the number of neurons is too small, the fault-tolerant ability of the network is poor. In general, the number of neurons does not exceed $20 .^{32}$ In this study, matlab cyclic structure was used to find the optimal number of neurons by comparing the root mean square error (RMSE) values of Elman networks with neurons 1-20. 
Fourth step. According to the optimal number of neurons in the hidden layer, the Elman model is constructed, and then the prediction and analysis can be made.

\section{Model comparison measures}

Three performance indexes, RMSE, mean absolute error (MAE) and mean absolute percentage error (MAPE) were used to assess the fitting and forecasting accuracy of two models. The smaller these three values are, the better the model is. Their expressions are as follows ${ }^{10}$ :

$$
\begin{aligned}
& R M S E=\sqrt{\frac{\sum_{t=1}^{n}\left(X_{t}-\hat{X}_{t}\right)^{2}}{n},} \\
& M A E=\frac{\sum_{t=1}^{n}\left|X_{t}-\hat{X}_{t}\right|}{n}, \\
& M A P E=\frac{\sum_{t=1}^{n} \frac{\left|X_{t}-\hat{X}_{t}\right|}{X_{t}} \times 100}{n},
\end{aligned}
$$

where, $\hat{X}_{t}$ is the simulating and forecasting values, $X_{t}$ is the actual values and $n$ is the number of observations.

\section{Statistical software}

All data analyses were conducted using Eviews7, matlab2015b, ArcMap V.10.1.

\section{RESULTS}

From January 2005 to December 2017, the number of reported TB cases was 141984 in Kashgar, Xinjiang, the average annual TB cases were 7888 and the average annual incidence was 191.18 per 100000 population. Figure 3 shows the time series graph of the TB incidence. It can be seen from the figure 3 that the curve of TB incidence has strong non-linear characteristics from 2005 to 2014, and the TB incidence from 2015 to 2017 was significantly higher than that of previous years.

The data of TB incidence from January 2005 to December 2017 were divided into two parts. The data from January 2005 to December 2016 were used to build model and the data from January 2017 to December 2017 were used to test the model.

\section{Establishment of the ARMA Model}

The ARMA modelling requires data stability, so, first of all, the stability of the modelling part of the data was verified by the ADF test. The results of the ADF test showed that $\mathrm{p}$ value was less than 0.05 (see table 1 ), which indicated

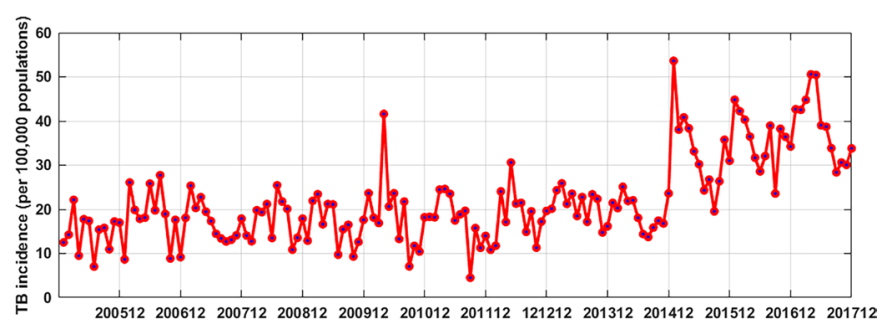

Figure 3 Graph of the tuberculosis (TB) incidence in Kashgar from January 2005 to December 2017. The curve of TB incidence shows strong non-linear characteristics from 2005 to 2014, and the TB incidence increases significantly from 2015 to 2017.
Table 1 The augmented Dickey-Fuller (ADF) test of the training data

\begin{tabular}{lrll}
\hline t-Statistics & & & P value \\
\hline ADF test statistic & & -3.47 & 0.01 \\
Test critical values & $1 \%$ level & -3.48 & \\
& $5 \%$ level & -2.88 & \\
& $10 \%$ level & -2.58 & \\
\hline
\end{tabular}

that the data were stable and could be directly used to build the model. Second, the ACF and the PACF graphs of the modelling data were plotted (see figure 4), it was obvious from figure 4 that the autocorrelation coefficients are trailing distribution and the partial correlation coefficients are almost a second-order truncated distribution, only at the lag 7, 8 and 9 , the correlation coefficients are a little large. Based on this situation, we considered establishing four models: AR (2) model, $\mathrm{AR}((1,2,7))$ model, AR $((1,2,8))$ model and AR $((1,2,9))$ model. The least square method was used to test the parameters of the four models. The results of the test were shown in table 2; we can see that, of the four models, only the AR (2) model and the AR $((1,2,8))$ model passed the parameter test. Comparing the two models, it was found that the AR $((1,2,8))$ model had smaller AIC and SC values, and the $\mathrm{R}^{2}$ value of the $\mathrm{AR}((1,2,8))$ model was larger than the $R^{2}$ value of the AR (2), so the AR (2) model was abandoned. Then, the residual analysis of the AR $((1,2,8))$ model was carried out, the autocorrelation and partial correlation coefficient of the residuals were almost all within two times SD, and only in the lag 5, 6, 12, they were beyond the range of two times standard deviation (see figure 5), which indicated that the $\operatorname{AR}((1,2,8))$ model could be used to roughly predict the TB incidence in Kashgar. We used the AR $((1,2,8))$ model to fit the TB incidence from September 2005 to December 2016, the fitting RMSE, MAE and MAPE were 6.15, 4.33 and 0.2858, respectively; we used the $\mathrm{AR}((1,2,8))$ model to predict the TB incidence from January 2017 to December 2017,
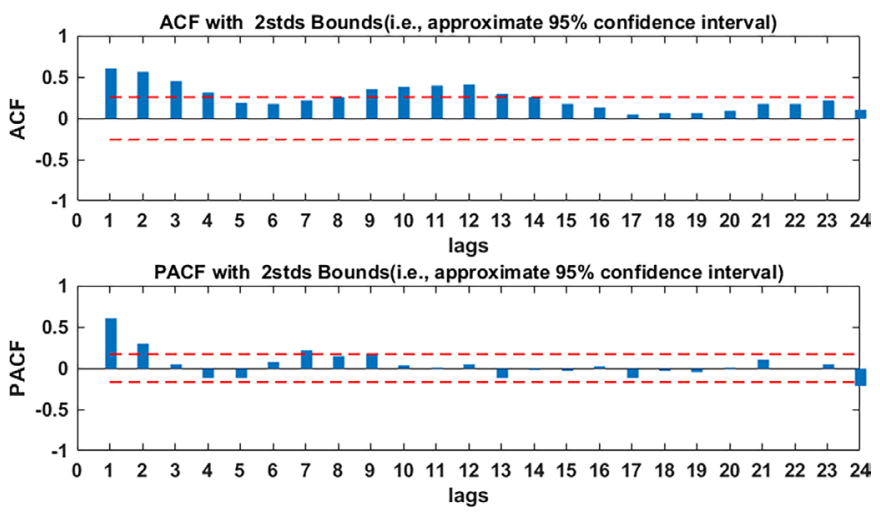

Figure 4 Autocorrelation function (ACF) and partial ACF (PACF) graphs of modelling data. As the delay of the lag order, the autocorrelation coefficients are trailing and the partial correlation coefficients are truncated, so it was deemed suitable to use the AR model. AR, autoregressive. 
Table 2 Parameter estimates of the tentative models with their Akaike information criterion (AIC) and Schwarz criterion (SC) values

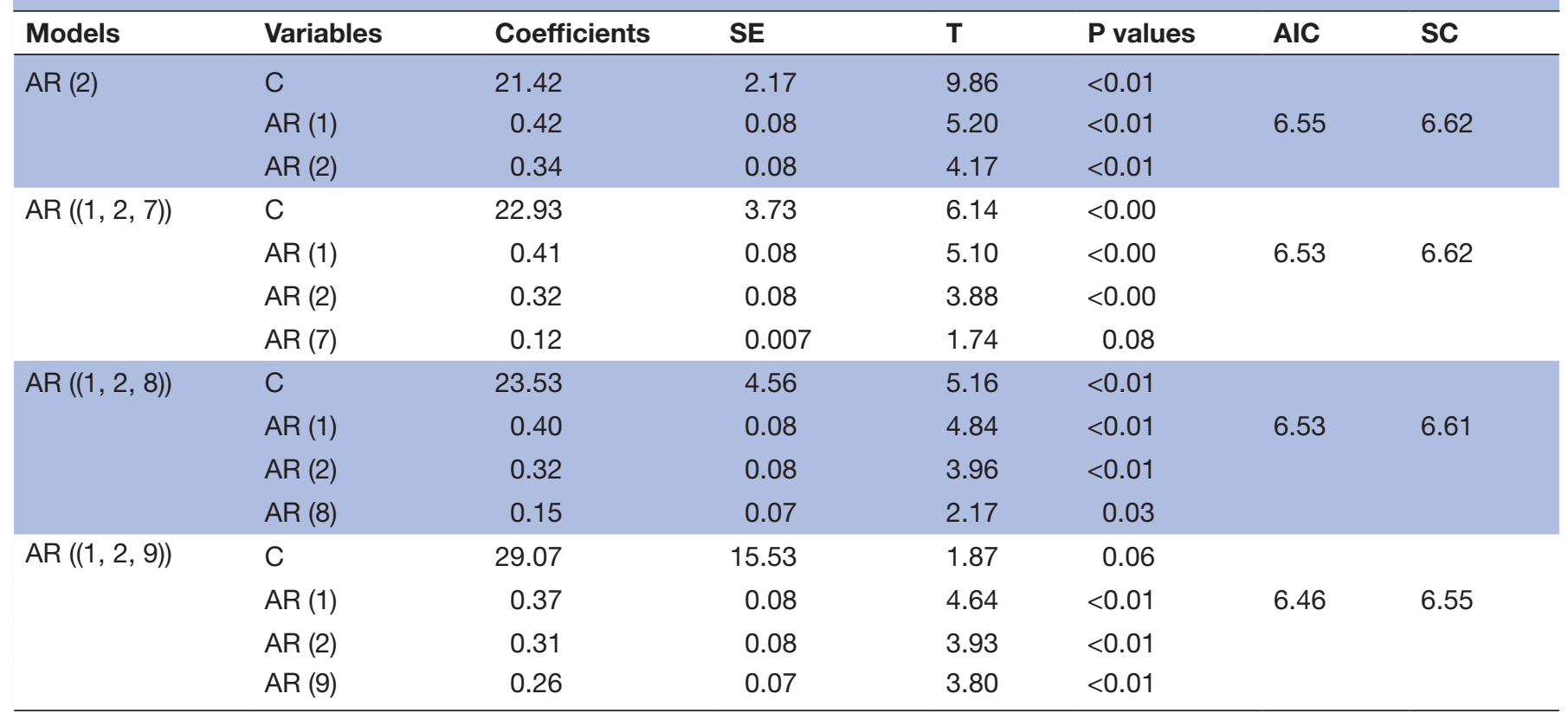

AR, autoregressive.

the prediction RMSE, MAE and MAPE were 10.88, 8.75 and 0.2029 , respectively.

\section{Establishment of the AR-Elman model}

In order to improve the prediction accuracy of the AR $((1,2,8))$ model, we tried to establish an AR $((1,2,8))$ Elman hybrid model. The fitting sequence of the AR ((1, $2,8)$ ) model was used as input variable, and the actual TB incidence was used as output variable. Due to a little similarity of the annual trend of TB incidence in Kashgar (see figure 3), therefore, we created 12 time-lagged variables as input features. Supposing that $x_{t}$ represented the TB incidence at time $t$, and then the input matrix and the output matrix of modelling data set used in this study were designed as follows $(\mathrm{N}=12)$
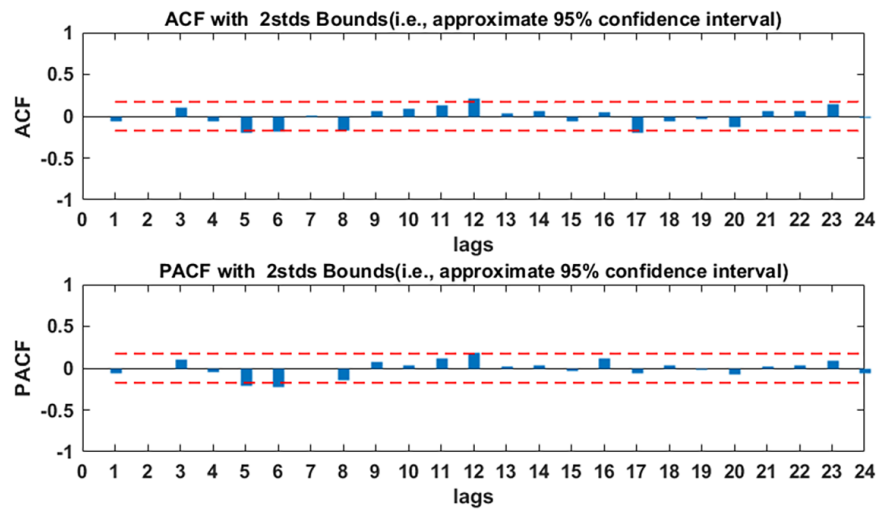

Figure 5 Autocorrelation function (ACF) and partial ACF (PACF) graphs of residuals of $A R((1,2,8))$ model.

Autocorrelation coefficients and partial correlation coefficients are almost in $95 \% \mathrm{Cl}$, so $\mathrm{AR}((1,2,8))$ model can extract the information of original data well. AR, autoregressive.

$$
\text { input matrix }=\begin{array}{cc}
x_{1} & x_{2} \ldots x_{i} \\
x_{2} & x_{3} \ldots x_{i+1}, \text { output matrix }=\left[x_{N+1} x_{N+2} \ldots\right. \\
x_{N} & x_{N+1} \ldots \ldots
\end{array}
$$$$
\left.x_{N+i}\right]
$$

We selected 12 as the number of input layers of AR-Elman network and one as the number of output layers representing the forecast value. By the matlab cyclic structure, we selected the optimal number of neurons between 1 and 20, and finally, we found when the number of neurons was 6 (see figure 6), the RMSE was the smallest, and the AR-Elman was optimal. We used the AR-Elman model to fit the training data, RMSE was 3.78, MAE was 3.38, MAPE was 0.1837 , and the $\mathrm{R}^{2}$ of the model was 0.83 ; we used the AR-Elman model to predict the TB incidence from January 2017 to December 2017, RMSE was 8.86, MAE was 7.29, and MAPE was 0.2006. The fitting curves of the $\operatorname{AR}((1,2,8))$ model and the AR-Elman model, and

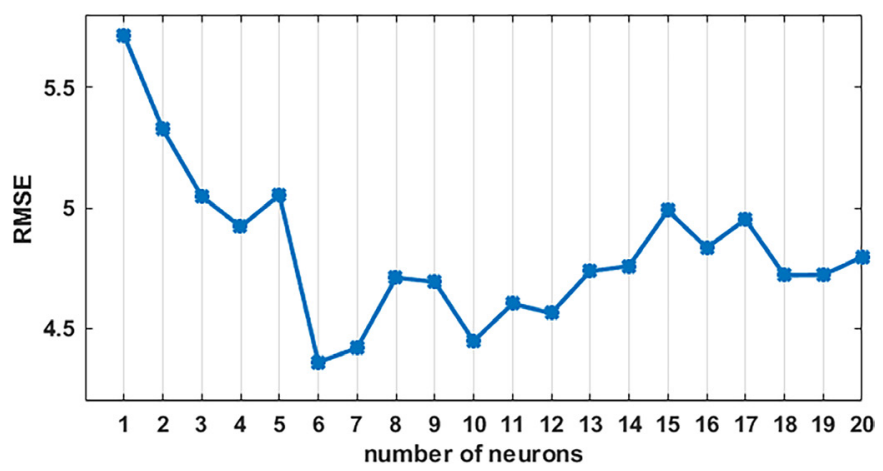

Figure 6 The numbers of neurons in AR-Elman model and the corresponding root mean square error (RMSE). When the number of neuron is 6 , the RMSE was the smallest, and the AR-Elman model fitting ability is the best. AR, autoregressive. 


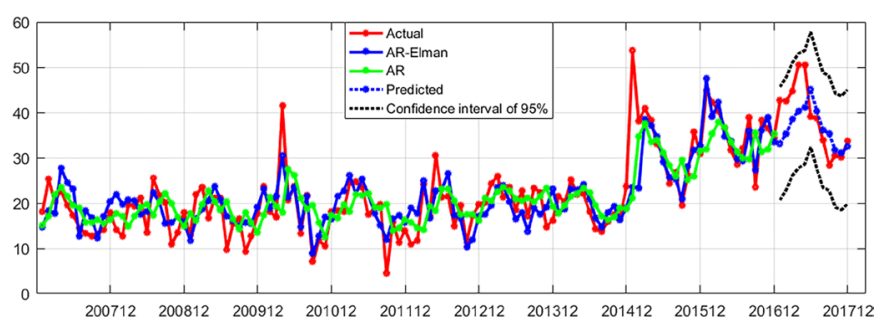

Figure 7 The fitting curves of the $\operatorname{AR}((1,2,8))$ model and the AR-Elman model, and the prediction curve of the ARElman model. The red line stands for the original tuberculosis incidence curve, the green line stands for $\operatorname{AR}((1,2,8))$ model fitting curve, and the blue line stands for AR-Elman model fitting curve. The blue dotted line stands for prediction curve of the AR-Elman model, the black dotted line stands for predicted curve of Cls. The fitting ability of AR-Elman hybrid model was slightly better than that of the single $A R((1,2,8))$. AR, autoregressive.

the prediction curve of the AR-Elman model are shown in figure 7. Comparison results of the $\operatorname{AR}((1,2,8))$ model and the AR-Elman model are shown in table 3, both the fitting RMSE, MAE and MAPE and the predicting RMSE, MAE and MAPE of the AR-Elman model are smaller than those of the single AR $((1,2,8))$ model, which indicated that the AR-Elman combined model established in this study was more suitable for predicting the TB incidence in Kashgar.

\section{DISCUSSION}

According to the WHO 2019 Global TB report, ${ }^{4}$ around the world, TB mortality was down about $3 \%$ every year, the incidence was down about $2 \%$ every year, $16 \%$ of TB patients died of the disease. ${ }^{4}$ But the rate of decline has not reached the pace of the 'stop TB Strategy Plan'. Therefore, it is necessary to strengthen the prevention and control of TB. In order to significantly narrow these gaps, greater progress must be made in a group of countries with a high burden of TB. The burden of TB in China ranks second in the world, and Xinjiang is the province with high incidence of ТВ in China, and Kashgar is the area with the high TB incidence in Xinjiang. Therefore, it was considered a high priority urgent to do a good job in the prevention and control of TB in Kashgar.

The prediction and early warning of infectious diseases is an important link in the prevention and control of infectious diseases. ${ }^{32-34}$ Therefore, this study carried out research from the point of view of prediction to explore an accurate prediction model and do the prediction analysis of TB incidence in Kashgar, so as to provide scientific reference for the prevention and control of the disease in this area. The Box-Jenkins method is a popular time series prediction method, this method has good prediction performance and high prediction accuracy; Elman Neural network can capture nonlinear information of time series data very well. In this study, the two methods were combined to study the prediction model of TB incidence in Kashgar.

Many studies have found that Box-Jenkins method has a good ability of fitting and forecasting. For stationary time series that do not contain seasonality, it is more suitable to use the ARMA model of the Box-Jenkins method to do prediction analysis,${ }^{35}$ for non-stationary time series of infectious diseases with obvious seasonality, it is more suitable to use the seasonal autoregressive integrated moving average (SARIMA) model of the Box-Jenkins method for prediction analysis. ${ }^{9-12}$ In our study, from figure 3, we could see that the seasonality of the TB incidence in Kashgar from 2005 to 2014 was not obvious, there was only a certain seasonality from 2015 to 2017, and we found that the time series of TB incidence was stable by the ADF unit root test, and the autocorrelation and partial correlation coefficients of modelling data at lag 12, 24 were not obviously large, therefore, for our research data, we used the ARMA model to do forecast analysis, and finally, we established the AR $((1,2,8))$ model of the Box-Jenkins method with its good performance in fitting and predicting the TB incidence of Kashgar in Xinjiang. In figure 3, we can also see that the time series of TB incidence has strong non-linear, Since the AR $((1,2,8))$ model we settled on mainly extracted the linear information of data, and knowing that the neural network can capture the nonlinear information of data well, we used the AR $((1,2$, 8) ) model and Elman neural network model to establish the AR-Elman hybrid model and improve the prediction accuracy of TB incidence rate in Kashgar. Many studies have found that the combination model can improve the accuracy of prediction: Wang $e t a l^{28}$ found that SARIMAnon-linear autoregressive network (NAR) hybrid model has an outstanding ability to improve the prediction accuracy relative to SARIMA model and NAR model when they were used to predict pertussis incidence in China. Li $e t a l^{27}$ found ARIMA-GRNN hybrid model was shown to be superior to the single ARIMA model in predicting the short-term TB incidence in the Chinese population.

Table 3 Comparison results of in-sample fitting and out-of-sample forecasting performance for the AR ((1, 2, 8)) model and the AR-Elman model

\begin{tabular}{|c|c|c|c|c|c|c|c|}
\hline \multirow[b]{2}{*}{ Models } & \multicolumn{3}{|c|}{ Fitted efficacy } & \multirow[b]{2}{*}{ Models } & \multicolumn{3}{|c|}{ Forecasted efficacy } \\
\hline & RMSE & MAE & MAPE & & RMSE & MAE & MAPE \\
\hline $\operatorname{AR}((1,2,8))$ & 6.15 & 4.33 & 0.2585 & $\operatorname{AR}((1,2,8))$ & 10.88 & 8.75 & 0.2029 \\
\hline
\end{tabular}

AR, autoregressive; MAE, mean absolute error; MAPE, mean absolute percentage error; RMSE, root mean square error. 
Our research was consistent with these literatures that our AR-Elman hybrid model was more accurate than the single AR $((1,2,8))$ model.

In the past few years, Xinjiang's economic development was relatively backward, medical resources were scarce, diagnosis and treatment were delayed, the continuous spread of TB has become a difficult problem in Xinjiang. In recent years, Xinjiang has introduced many new policies to increase investment in TB prevention and control, and the relevant departments of disease prevention and control in Xinjiang have also done a lot of effective work, which has helped to control the rapid increase of the TB incidence in Xinjiang. In order to do a good job in the prevention and control of TB in Xinjiang, many departments need to make joint efforts. Our research was mainly to build a high-precision prediction model to help early warning and prediction analysis of TB in Kashgar. Finally, we established the AR-Elman hybrid model, which had high fitting and prediction accuracy of TB incidence in Kashgar, Xinjiang.

Our study found that Box-Jenkins and Elman neural network hybrid method is an effective method for predicting the incidence of TB in Kashgar, it can provide a scientific reference for prediction analysis of TB incidence. However, our study also has some limitations: our method is only suitable for short-term prediction, longterm prediction performance will decline, for two main reasons: first, our model was based on historical data characteristics; second, climatic factors, environmental factors, demographic factors and political issues may have certain impacts on the rate of change of TB incidence. Therefore, if the established model becomes old and researchers want to obtain more accurate prediction results, the model parameters will need to be adjusted, the model updated based on the new modelling sample data, and then the prediction analysis redone.

\section{CONCLUSIONS}

Kashgar has a very high TB incidence, in order to provide some help for the prevention and control of this disease, the prediction problem of the TB incidence was studied. First, a single AR $((1,2,8))$ prediction model was established by using Box-Jenkins method, with good fitting and prediction performance. Second, in order to improve the prediction accuracy of the single AR $((1,2,8))$ model, we used the single AR $((1,2,8))$ and the Elman neural network with its strong ability to capture nonlinear information to establish AR-Elman hybrid model. The fitting and prediction accuracy of the hybrid model was higher than that of the single AR $((1,2,8))$ model. The AR-Elman hybrid model can provide a scientific reference for predicting and warning of the TB incidence in Kashgar, Xinjiang.

Acknowledgements We would like to thank peer reviewers for their carefully revising our manuscript and for their very useful comments, and we would like to thank Lloyd Murat Maxson, a native English-speaking colleague, for his great help with the quality of the English.
Contributors $\mathrm{YZ}$ and $\mathrm{XZ}$ analysed the data and wrote the manuscript. $\mathrm{XZ}, \mathrm{XW}, \mathrm{KW}$ and $Y C$ wrote and revised the manuscript. All authors read and approved the final manuscript.

Funding This work was supported by Major projects of science and technology in Xinjiang Autonomous Region (grant no.2017A03006), China, and National Natural Science Foundation Project of China (grant no. 72064036, 82060609), and State Key Laboratory of Pathogenesis, Prevention and Treatment of High Incidence Diseases in Central Asia Fund (grant no. SKL-HIDCA-2020-9).

Map disclaimer The depiction of boundaries on the map(s) in this article does not imply the expression of any opinion whatsoever on the part of BMJ (or any member of its group) concerning the legal status of any country, territory, jurisdiction or area or of its authorities. The map(s) are provided without any warranty of any kind, either express or implied.

Competing interests None declared.

Patient consent for publication Not required.

Ethics approval Since no primary data collection was undertaken, no patient or public was involved; no formal ethical assessment or informed consent was required.

Provenance and peer review Not commissioned; externally peer reviewed.

Data availability statement Data are available on reasonable request. The data used in this study are available from the corresponding authors on reasonable request.

Open access This is an open access article distributed in accordance with the Creative Commons Attribution Non Commercial (CC BY-NC 4.0) license, which permits others to distribute, remix, adapt, build upon this work non-commercially, and license their derivative works on different terms, provided the original work is properly cited, appropriate credit is given, any changes made indicated, and the use is non-commercial. See: http://creativecommons.org/licenses/by-nc/4.0/.

ORCID iD

Yanling Zheng http://orcid.org/0000-0003-4810-5488

\section{REFERENCES}

1 Kemal J, Sibhat B, Abraham A, et al. Bovine tuberculosis in eastern Ethiopia: prevalence, risk factors and its public health importance. BMC Infect Dis 2019;19:39.

2 Tilahun M, Ameni G, Desta K, et al. Molecular epidemiology and drug sensitivity pattern of Mycobacterium tuberculosis strains isolated from pulmonary tuberculosis patients in and around Ambo town, central Ethiopia. PLoS One 2018;13:e0193083.

3 Reta A, Simachew A. The role of private health sector for tuberculosis control in Debre Markos town, Northwest Ethiopia. Adv Med 2018;2018:1-8.

4 WHO. Global tuberculosis report, 2019. Available: https://www.who. int/tb/publications/global_report/en/ [Accessed 07 Oct 2019].

5 Zhao Y-F, Shou M-H, Wang Z-X. Prediction of the number of patients infected with COVID-19 based on rolling grey Verhulst models. Int $J$ Environ Res Public Health 2020;17:4582.

6 Guan P, Wu W, Huang D. Trends of reported human brucellosis cases in mainland China from 2007 to 2017: an exponential smoothing time series analysis. Environ Health Prev Med 2018;23:23.

7 Zhang Y-Q, Li X-X, Li W-B, et al. Analysis and predication of tuberculosis registration rates in Henan Province, China: an exponential smoothing model study. Infect Dis Poverty 2020;9:123.

8 Martínez-Bello DA, López-Quílez A, Torres-Prieto A. Bayesian dynamic modeling of time series of dengue disease case counts. PLoS Negl Trop Dis 2017;11:e0005696.

9 Wang Y, Xu C, Zhang S, et al. Temporal trends analysis of human brucellosis incidence in mainland China from 2004 to 2018. Sci Rep 2018;8:15901.

10 Wang Y, Xu C, Li Y, et al. An advanced data-driven hybrid model of SARIMA-NNNAR for tuberculosis incidence time series forecasting in Qinghai Province, China. Infect Drug Resist 2020;13:867-80.

11 Aryee G, Kwarteng E, Essuman R, et al. Estimating the incidence of tuberculosis cases reported at a tertiary hospital in Ghana: a time series model approach. BMC Public Health 2018;18:1292.

12 Tohidinik HR, Mohebali M, Mansournia MA, et al. Forecasting zoonotic cutaneous leishmaniasis using Meteorological factors in eastern Fars Province, Iran: a SARIMA analysis. Trop Med Int Health 2018;23:860-9. 
13 Ouedraogo B, Inoue Y, Kambiré A, et al. Spatio-Temporal dynamic of malaria in Ouagadougou, Burkina Faso, 2011-2015. Malar J 2018;17:138.

14 Wagenaar BH, Augusto O, Beste J, et al. The 2014-2015 Ebola virus disease outbreak and primary healthcare delivery in Liberia: timeseries analyses for 2010-2016. PLoS Med 2018;15:e1002508.

15 Liu S, Chen J, Wang J, et al. Predicting the outbreak of hand, foot, and mouth disease in Nanjing, China: a time-series model based on weather variability. Int J Biometeorol 2018;62:565-74

16 Anokye R, Acheampong E, Owusu I, et al. Time series analysis of malaria in Kumasi: using ARIMA models to forecast future incidence. Cogent Soc Sci 2018;4:1461544.

17 Wang Y-W, Shen Z-Z, Jiang Y. Comparison of autoregressive integrated moving average model and generalised regression neural network model for prediction of haemorrhagic fever with renal syndrome in China: a time-series study. BMJ Open 2019;9:e025773.

18 Guo W-L, Geng J, Zhan Y, et al. Forecasting and predicting intussusception in children younger than 48 months in Suzhou using a seasonal autoregressive integrated moving average model. BMJ Open 2019;9:e024712.

19 Gabriel AFB, Alencar AP, Miraglia SGEK. Dengue outbreaks: unpredictable incidence time series. Epidemiol Infect 2019;147:e116.

20 Tian CW, Wang H, Luo XM. Time-Series modelling and forecasting of hand, foot and mouth disease cases in China from 2008 to 2018 Epidemiol Infect 2019;147:e82.

21 Juang W-C, Huang S-J, Huang F-D, et al. Application of time series analysis in modelling and forecasting emergency department visits in a medical centre in southern Taiwan. BMJ Open 2017;7:e018628.

22 Yu C, Li Y, Zhang M. An improved wavelet transform using singular spectrum analysis for wind speed forecasting based on Elman neural network. Energy Convers Manag 2017;148:895-904.

23 Huang Y, Shen L. Elman Neural Network Optimized by Firefly Algorithm for Forecasting China's Carbon Dioxide Emissions.
Communications in Computer and Information Science 2018;951:36-47.

24 Alkhasawneh MS. Hybrid cascade forward neural network with Elman neural network for disease prediction. Arab J Sci Eng 2019;44:9209-20.

25 Mehrgini B, Izadi $\mathrm{H}$, Memarian $\mathrm{H}$. Shear wave velocity prediction using Elman artificial neural network. Carbonates \& Evaporites 2017;6:1-11.

26 Khalaf M, Hussain AJ, Keight R, et al. Machine learning approaches to the application of disease modifying therapy for sickle cell using classification models. Neurocomputing 2017;228:154-64.

$27 \mathrm{Li}$ Z, Wang Z, Song H, et al. Application of a hybrid model in predicting the incidence of tuberculosis in a Chinese population. Infect Drug Resist 2019;12:1011-20.

28 Wang $Y, X u$ C, Wang Z, et al. Time series modeling of pertussis incidence in China from 2004 to 2018 with a novel wavelet based SARIMA-NAR hybrid model. PLoS One 2018;13:e0208404.

29 Wang Y, Xu C, Zhang S, et al. Temporal trends analysis of tuberculosis morbidity in mainland China from 1997 to 2025 using a new SARIMA-NARNNX hybrid model. BMJ Open 2019;9:e024409.

30 Box GE, Jenkins GM. Time series analysis: forecasting and control. Oakland, California: Holden-Day, 1976: 31. 238-42.

31 Cheng M. The principle and example of Matlab neural network Tsinghua University Press, 2013.

32 Huppert A, Katriel G. Mathematical modelling and prediction in infectious disease epidemiology. Clin Microbiol Infect 2013;19:999-1005.

33 Wearing HJ, Rohani P, Keeling MJ. Appropriate models for the management of infectious diseases. PLoS Med 2005;2:e174.

34 Neuberger A, Paul M, Nizar A, et al. Modelling in infectious diseases: between haphazard and hazard Clin Microbiol Infect 2013:19:993-8.

35 Tipirneni-Sajja A, Krafft AJ, Loeffler RB, et al. Autoregressive moving average modeling for hepatic iron quantification in the presence of fat. J Magn Reson Imaging 2019;50:1620-32. 